\title{
Improving the in vitro penetration of niacinamide using span 60-based niosomal system in gel formulation
}

\author{
Rise Desnita, Sri Luliana, Zainab* \\ Departement of Pharmacy, Medical Faculty, Tanjungpura University \\ Jl. Prof. Dr. H. Hadari Nawawi 78124
}

Submitted: 30-10-2917

Reviewed: $17-04-2018$

Accepted: 02-11-2108

\begin{abstract}
Niacinamide is one of the components in cosmeceutical products that have been reported to offer benefits for skin pigmentation, acne, and wrinkles treatment. However, the dermal penetration of niacinamide is poor. The carrier system that can transport the compound through the stratum corneum layer is the niosomal system prepared using Span 60. This study aimed to determine at which concentration Span 60 could entrap niacinamide optimally and the ability of the Span 60-based niosomal system to increase in vitro penetration of niacinamide in gel preparation. The nicotinamideloaded niosomes were formulated with various concentrations of Span 60, namely (A) $100 \mu \mathrm{mol}$, (B) $150 \mu \mathrm{mol}$, and (C) $200 \mu \mathrm{mol}$. The entrapment efficiency testing was conducted using dialysis membranes. The gel was prepared with two formulas i.e., niacinamide-loaded niosome gel and niacinamide gel (without niosomes) and tested for diffusion using the flow-through Franz diffusion cell. The results showed that Formula A provided the most optimum entrapment efficiency of $99.03 \pm 0.026 \%$. Meanwhile, the results of the in vitro skin penetration studies for eight (8) hours indicated that the niosomal formulation prepared with Span 60 could increase dermal penetration. The cumulative percentage of niacinamide-loaded niosomes penetrating the skin was $82.87 \pm 1.6932 \%$ or higher than that of niacinamide gel without niosomal system, i.e., about $70.27 \pm 5.3212 \%$.
\end{abstract}

Keywords: niacinamide, gel, niosome, Span 60, in vitro penetration

\footnotetext{
*Corresponding author:

Zainab

Departement of Pharmacy, Medical Faculty, Tanjungpura University

J1. Prof. Dr. H. Hadari Nawawi 78124

Email: zainab74412@gmail.com
} 


\section{INTRODUCTION}

Niacinamide is a class of vitamins and one of the components in cosmeceutical products that has been reported to have beneficial effects on skin in the treatment of skin pigmentation, acne, and wrinkles (Griffiths, 1995). Topical use of niacinamide as a gel or cream is not effective enough because it barely permeates the stratum corneum layer of the skin (Sara et al., 2008). Therefore, a proper carrier system is needed to deliver the compound across this layer, for instance, the niosomal system.

Niosomes have been related to an increase in the dermal penetration of some compounds. They can entrap drug ingredients and make them easily diffuse through the lipid bilayer and, then, the stratum corneum (Jigar et al., 2011). The surfactant used in the niosomal system is sorbitan monostearate (Span 60). As a constituent of this system, Span 60 has been used for delivering various active substances, including flutamide and dexamethasone (Navya et al., 2014; Mavaddati et al., 2015). However, the use of Span 60-based niosomal system for niacinamide has never been done before.

Based on these considerations, this research aimed to determine at which concentration Span 60 could entrap niacinamide in the niosomal system optimally to increase the penetration of niacinamideloaded niosomes.

\section{MATERIALS AND METHODS \\ Materials}

The materials used in this study included niacinamide, Span 60 (Sigma-Aldrich), cholesterol (Sigma-Aldrich), chloroform (Merck), distilled water, Viscolam MAC 10, Dimethyl dimethylol hydantoin (Sharon), triethanolamine (Clorogreen), dialysis tubing cellulose membrane type d9777-100 FT cut-off 14000 (Carolina TM), sodium dihydrogen phosphate, disodium hydrogen phosphate, sodium hydroxide, and shed snake skin (Phyton reticulatus) as the membrane.

\section{Methods}

\section{Niosomes preparation}

The niosomes were prepared with the classic method Thin-Film Hydration. The Span 60 and cholesterol were dissolved in $10 \mathrm{~mL}$ of chloroform in a $100 \mathrm{~mL}$ round-bottom flask. Then, the solvent was removed using a rotary evaporator at $40 \pm 2^{0} \mathrm{C}$ and $150 \mathrm{rpm}$, forming a thin layer on the wall of the rotating flask. The flask was then placed in the desiccator and left for 24 hours (Apridamayanti $e t$ al., 2016). Afterward, the thin layer was hydrated with $10 \mathrm{~mL}$ of niacinamide solution using a rotary evaporator at $60 \pm 2^{0} \mathrm{C}$ and $150 \mathrm{rpm}$ until all of the thin layers on the wall of the rotating flask were removed. The niacinamide solution was prepared by dissolving $100 \mathrm{mg}$ of niacinamide in $10 \mathrm{~mL}$ of distilled water. Furthermore, the particle size was reduced using a magnetic stirrer at 1,000 rpm for 1 hour. The formulas of the niosomes are shown in Table I.

Table I. The formulas of the niacinamide-loaded niosomes

\begin{tabular}{cccc}
\hline Materials & Formula A & Formula B & Formula C \\
\hline Niacinamide $(\mathrm{mg} / \mathrm{mL})$ & 10 & 10 & 10 \\
Span $60(\mu \mathrm{mol})$ & 100 & 150 & 200 \\
Cholesterol $(\mu \mathrm{mol})$ & 20 & 30 & 40 \\
\hline
\end{tabular}

\section{Entrapment efficiency testing}

The entrapment efficiency was determined by the dialysis method. The receptor medium used in this analysis was $200 \mathrm{~mL}$ of distilled water. The test was carried out for 3 hours. The samples were then analyzed using a UV-Vis spectrophotometer at a wavelength of $261.5 \mathrm{~nm}$. The entrapment efficiency was calculated with the following equation (Eq. 1) (Rahman et al., 2011). 
where:

$$
\mathrm{EE}=\frac{Q t-Q C}{Q t} X 100 \%
$$

$\mathrm{EE}=$ Entrapment efficiency

$\mathrm{Qt}=$ The amount of niacinamides added to the formula

$\mathrm{Qc}=$ The amount of niacinamides in the receptor medium (not entrapped)

\section{Morphological observation and vesicle size measurement}

The morphology of the formulated niosomes was observed under an optical microscope (Zeiss Primo Star Axio CamERc5s). The size of the vesicles was measured using Particle Size Analyzer (PSA) (Beckman Coulter).

\section{Gel formulation}

The niacinamide gel was prepared by dissolving niacinamide and DMDM hydantoin in distilled water. Afterward, the solution was added with viscolam MAC 10, stirred until homogeneous, added with TEA, and then stirred gently continuously until the desired gel preparation was formed. Meanwhile, the niacinamide niosomal gel was made by adding DMDM hydantoin to the niacinamide-loaded niosomes and then mixing it with a gel base thickened with Viscolam MAC 10 using a low-speed stirrer.

Table II. The formulations of the niacinamide niosomal gel and niacinamide gel

\begin{tabular}{ccc}
\hline Materials & Formula I & Formula II \\
\hline Niacinamide-loaded Niosomes (\%) & 1 & - \\
Niacinamide (\%) & - & 1 \\
DMDM Hydantoin (\%) & 0.06 & 0.06 \\
Viscolam MAC 10 (\%) & 8 & 8 \\
TEA & 4 drops & 4 drops \\
Distilled water(g) & to 10 & to 10 \\
\hline
\end{tabular}

\section{Diffusion test}

The diffusion test was performed in vitro using the flow-through Franz diffusion cell method on the shed snake skin as the membrane. The receptor compartment was filled with a solution of phosphate buffer $\mathrm{pH} 7.4,50 \mathrm{~mL}$, which was maintained at approximately $37 \pm 0.5^{\circ} \mathrm{C}$. The membrane was then placed between the donor and the receptor compartments with the horn layer facing up. Around $200 \mathrm{mg}$ sample was applied to the surface of the membrane. Then, $5 \mathrm{~mL}$ sample was collected from the receptor compartment after $0.5,1,2,3,4,5,6,7$, and 8 hours using a volume pipette and was replaced immediately with the same volume of a solution of phosphate buffer $\mathrm{pH} 7.4$ at the same temperature. Afterward, the absorption was measured at a wavelength of $261.5 \mathrm{~nm}$ with a UV-Vis spectrophotometer. The test was replicated three times. The cumulative amount of the penetrated niacinamide was calculated with the following equation (Eq. 2):

$$
\% \text { Diffusion }=\frac{\text { the amount of active substancein the receptor compartment }}{\text { the amount of active substance in the formula }}
$$

\section{Data Analysis}

The data were analyzed in a computer program, SPSS 21.0 for Windows. The statistical tests included the One-Way ANOVA for the entrapment efficiency data and the independent sample ttest for the diffusion test results. 


\section{Results and Discussion \\ The formulated niosomes}

The formulated niosomes had the typical odor of Span 60 and milky white color. The formulation of niosomes in this study employed the Thin-Layer Hydration because of its most common, easy, and simple use and the availability of equipment in the laboratory. The niosomal vesicles were formed spontaneously when the thin layer on the wall of the rotating flask was hydrated with a water phase, i.e., distilled water. Because niacinamide is hydrophilic, it was added to the water phase during the hydration process. The temperature of the hydration affects the shape and size of the formulated niosomes. The temperature used to create niosomes is normally above the temperature of the gel-to-liquid phase transition of the niosomal system (Akhilesh et al., 2012). The temperature during the transition from the gel phase to the liquid phase of Span 60 is $56-58^{\circ} \mathrm{C}$ (Kumar et al., 2011). Therefore, the hydration process of each niosome formula used a rotary evaporator at a temperature of $60^{\circ} \mathrm{C}$.

\section{Entrapment efficiency}

The entrapment efficiency can be interpreted as a portion of active substances caught by niosomes (Seleci et al., 2016). The calculation results of the entrapment efficiency can be seen in Table III.

Table III. The percentage of entrapment efficiency

\begin{tabular}{ccc}
\hline Formula & Span 60:Cholesterol $(\boldsymbol{\mu m o l})$ & Entrapment efficiency $(\boldsymbol{\%}) \pm$ SD \\
\hline A & $100: 20$ & $99.03 \pm 0.0264$ \\
B & $150: 30$ & $99.04 \pm 0.0596$ \\
C & $200: 40$ & $99.13 \pm 0.0638$ \\
\hline
\end{tabular}

The table illustrates that the entrapment efficiency of niacinamide enhances with an increase in the concentration of Span 60 and cholesterol in the formula. Formula $C$ showed the highest entrapment efficiency, i.e., $99.13 \pm 0.0638 \%$. This result is in line with the previous study, which states that increasing the concentration of the surfactant affects the entrapment efficiency; a higher surfactant level leads to an increase in drug absorption efficiency and, at the same time, a decrease in drug leakage (Salih et al., 2013).

The significance values obtained from the One-Way ANOVA on the SPSS program were greater than 0.05 ( $p>0.05$ ), indicating the absence of significant differences between the three formulas. Therefore, Formula A was concluded as the one that provided optimum drug entrapment because the resultant entrapment efficiency was significantly different from the other niosome formulas that had a higher concentration of Span 60. Besides, the use of Formula A can also minimize the amount of the materials used in the future formulation.

\section{The morphology and vesicle size of niacinamide-loaded niosomes}

The vesicles of the niacinamide-loaded niosomes had a round shape. The results of the morphological observations of the formulated niosomes can be seen in Figure 1. Using the ICLE Part Size Analyzer (Beckman Coulter), this research found that the vesicles of the formulated niosomes had an average size of $5,197.6 \mathrm{~nm}$. Because the size was $>0.05 \mu \mathrm{m}$, these niosomes were categorized as having multilamellar vesicles (Biju et al., 2006). 


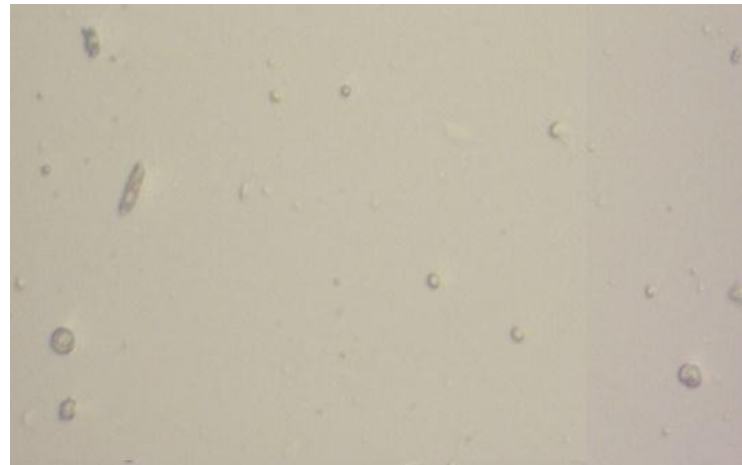

Figure 1. The morphology of the niosomes prepared with formula A (magnification 40x)

\section{Diffusion test results}

The diffusion test aimed to determine the amount of niacinamide that penetrated the skin during a specified time interval from the niacinamide gel and the niacinamide niosomal gel. The niosomal gel used in the diffusion tests was the one prepared with the niosome formula that produced optimum entrapment efficiency, i.e., $100 \mu \mathrm{mol}$ (Formula A). The results of the diffusion test can be seen in Figure 2.

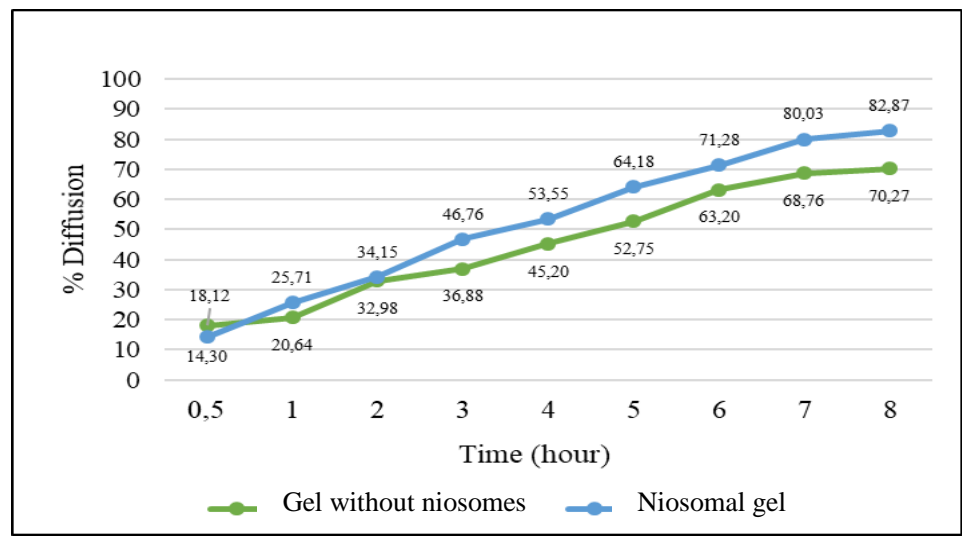

Figure 2. The diffusion test results

The cumulative percentages of niacinamide released from the niacinamide gel preparation (without niosomes) and the niacinamide-contained niosomal gel that penetrated the shed snake skin for 8 hours were $70.27 \pm 5.3212 \%$ and $82.87 \pm 1.6932 \%$, respectively. The results showed that the latter resulted in a higher amount of penetrated niacinamide than the former preparation. These data were analyzed statistically using the independent sample t-test, which resulted in a significance value of smaller than 0.05 ( $\mathrm{p}<0.05)$, indicating a significant difference between the \% diffusions of both gel preparations. When compared with niacinamide gel without niosomes, the presence of niosomal system dispersed in niacinamide gel preparations can increase the penetration of niacinamide through the stratum corneum.

\section{CONCLUSION}

This research concluded that the optimum entrapment efficiency was found in niacinamide niosomal gel prepared with $100 \mu \mathrm{mol}$ of Span 60 (\% entrapment efficiency $=99.03 \pm 0.0264 \%$ ). The use of Span 60 as a constituent of niacinamide-loaded niosomes can increase the dermal penetration of niacinamide with a cumulative diffusion of $82.87 \pm 1.6932 \%$ during the 8 -hour observation, which is higher than in niacinamide gel without niosomes i.e., $70.27 \pm 5.3212 \%$. 


\section{ACKNOWLEDGMENT}

Authors would like to express their gratitude to the Technology Laboratory (Labtek) VII, ITB Pharmacy School for the laboratory facilities provided during the research.

\section{REFERENCES}

Akhilesh, D., Bini, KB., Kamath, JV., 2012. Review on Span-60 based non-ionic surfactant vesicles (niosomes) as novel drug delivery, IJRPBS, 3(1): 6-12.

Apridamayanti, P., Listiyana, N., Desnita, R., 2016. Formulation vitamin c using niosomes system span 80 in gel for increase stability and penetration in vitro, Int J PharmTech Res, 9(2): 81-88.

Biju, S.S., Talegaonkar, S., Mishra, P.R., Khar, R.K., 2006, Vesicular system: an overview, Ind J Pharm Sci, 68(2): 141-153.

Griffiths, C.E.M., 1995. Nicotinamide $4 \%$ gel for the treatment of inflammatory acne vulgaris. $J$ Dermatolog Treat, 6: 8-10.

Jigar, V., Gajjar, V., Gediya, T., Christian, V., Upadhyay, U., 2011. Formulation and characterization of topical gel of erythromycin entrapped into niosomes, Int J PharmTech Res, 3(3): 1714-1718.

Kumar, G.P., Rajeshwarrao, 2011, Nonionic surfactants vesicular systems for effective drug delivery: an overview, Acta Pharmaceutica Sinica B, 1(4): 208-219.

Mavaddati, M.A., Moztarzadeh, F., Baghbani, F., 2015. Effect of formulation and processing variables on dexamethasone entrapment and release of niosomes, J Cluster Sci, 26(6): 2065-2078.

Navya, M.N., Parthiban, S., Senthilkumar, G.P., Mani, T.T., 2014. The effect of Span 60 in the formulation of niosomes containing flutamide, Int J Res Pharm Nano Sci, 3(5): 444- 449.

Rahman, L., Ismail, L., Wahyudin, E., 2011. Kapasitas jerap niosom terhadap ketoprofen dan prediksi penggunaan transdermal, Majalah Farmasi Indonesia, (2): 85-91.

Salih, O.S., Samein, L.H., Ali, W.K., 2013. Formulation and in vitro evaluation of rosuvastatin calcium niosomes, Int J Pharm Pharm Sci, 5(4): 525-535.

Sara, N., Franca, Z., Stefania, B., Ruggero, B., Patrizia, S., 2008. Association of nicotinamide with parabens: effect on solubility, partition and transdermal permeation, Eur J Pharm. Biopharm, 69(2): 613-621.

Seleci, D.A., Seleci, M., Walter, J.G., Stahl, F., Scheper, T., 2016. Niosomes as nanoparticular drug carriers: fundamentals and recent applications, Journal of Nanomaterials, 2016(7372306):1-3. 